\title{
Les médias contre la mémoire ?
}

Au cœur de nos sociétés dites "hypermédiatisées ", les médias se sont imposés comme le lieu par excellence où se diffusent et se construisent les perceptions, les représentations et les imaginaires qui forment et informent les rapports entre social et politique. Or, s'ils engagent le devenir des sociétés, ces rapports interrogent fondamentalement la relation au passé, inlassablement reconstruit pour dessiner le présent. Médias et mémoires ont ainsi partie liée, quitte à reconnaître que celle-ci soit marquée au sceau du manque ou du paradoxe.

Nombreux en effet sont les auteurs qui tendent à opposer médias et mémoires, communication et transmission, connaissance et souvenir. L'ère médiatique serait celle de l'éphémère, de l'immédiat, du flux paradoxalement figé dans une sorte de présent perpétuel, radicalement opposée au temps long et à l'enracinement dans le passé. Les médias ne véhiculeraient ainsi plus qu'une «mémoire morte », caractérisée par la pauvreté de son contenu et sa déconnexion du souvenir'. Dans le meilleur des cas ils dénatureraient la mémoire en la coupant de son référent, en l'arrachant à la terre - en la déterritorialisant - au profit d'une mémoire "évanescente, irréelle " ${ }^{2}$; au pire ils signeraient la perte de toute mémoire, que ce soit par manque ou par saturation, amnésie ou hypermnésie ${ }^{3}$. Dans les deux cas les médias, dans leur version dominante et globalisante, sont vus comme accompagnant et renforçant la perte du lien social authentique au profit d'un lien qui ne lierait plus, d'une communication sans contenu, signe de la dissolution des identités groupales fondées sur le souvenir partagé d'un passé commun.

Si l'on peut souscrire partiellement à ces affirmations, ces critiques adressées aux médias en matière de mémoires - car il s'agit bien de critiques - nous semblent révélatrices, bien plus que d'une rupture, d'un lien fort entre les deux. Pour le dire autrement, le fait même de

\footnotetext{
'À ce sujet, voir par exemple VALLIN Pierre, "Mémoire ou communication », Quaderni, n 13-14, "Territoire et communication », printemps 1991, p. 70. ${ }^{2}$ lbid.

${ }^{3}$ Voir notamment BouRASSA Renée, «Enjeux de la mémoire : entre hypermnésie et oubli », in CASSOU-NoGUÈz Pierre, LARSONNEUR Claire et RÉGNAULT Arnaud (dir), Le sujet digital: l'hypermnésie, Paris, Presse du Réel (à paraître, 2014), disponible à l'adresse suivante : http://www.academia.edu/ [consultée le 23 octobre 2014]
} 
critiquer le rôle des médias dans les transformations qui affectent les régimes mémoriels contemporains - c'est-à-dire les modes de constitution, de transmission et de configuration des mémoires, entre autres, ainsi que les productions stabilisées de mémoire publique officielle par des politiques mémorielles ${ }^{4}$ - revient paradoxalement à leur attribuer un rôle dans la construction mémorielle et à définir en creux, de manière négative, ce que devrait être ce rôle.

Ainsi la perspective adoptée dans ce numéro suivra-t-elle quelques-uns des nombreux fils qui tissent les rapports entre médias et mémoires, plutôt que de partir du postulat de leur incompatibilité. Ce dernier postulat nous semble en effet relever d'une vision pessimiste qui reviendrait à prendre acte d'une évolution dont rien ne prouve l'achèvement (consommé ou à venir) et qui oblitère dans un même geste les logiques de résistance, de changement; les forces " alternatives " - par opposition aux forces dominantes - qui, pour être marginales, n'en sont pas moins multiples et vives.

Alors nous faut-il examiner précisément ce rôle des médias, ce qu'ils "font " aux mémoires, à ses régimes de (re)production et de diffusion; le décrire par des analyses concrètes, qui plongent au cœur de la matière même de leurs rapports; c'est du moins le parti pris par ce numéro. Ainsi les contributions réunies ici se fondent-elles toutes sur des études de corpus, issus de médias de masse aussi bien que de médias à diffusion plus restreinte; qu'il s'agisse d'articles de presse, de productions cinématographiques, de documents numériques (sites web et pages de réseaux sociaux) ou encore de littérature scientifique. Au terme de ces analyses aux méthodes et aux objets très divers, et seulement alors, nous tâcherons de déterminer leurs points communs, ou plus précisément d'examiner la dimension normative qui y affleure, de manière à dégager quelques-uns des principes qui sont censés régir les rapports entre médias et mémoires collectives et des questionnements qui traversent le champ de ces études.

Nous espérons ainsi proposer quelques repères, quelques critères pour évaluer ces relations entre médias et mémoires collectives, notamment tangibles à travers les critiques plus ou moins explicites adressées aux médias dans les textes rassemblés ici. Nous interrogerons également les dimensions politiques de ces rapports, questionnement qui travaille à des degrés divers l'ensemble des contributions à ce numéro ; qu'il s'agisse des politiques renvoyant aux actions et déci-

${ }^{4}$ Johann Michel définit les politiques mémorielles comme l' «ensemble des interventions des acteurs publics visant à produire et à imposer une mémoire publique officielle à la société à la faveur du monopole d'instruments d'action publique ». MiCHEL Johann, Gouverner les mémoires. Les politiques mémorielles en France, Paris, Presses Universitaires de France, 2010, pp. 16-20. 
sions adoptées par des autorités politiques; de la politique entendue comme rapports de force entre les acteurs ; ou du politique concernant plus largement les règles du "vivre ensemble ".

En particulier, "Médias en jeu, enjeux de mémoires " s'articule autour de deux axes, ou deux points de fuite questionnant les rapports entre médias et mémoires collectives, et qui pointent précisément vers ces enjeux politiques que nous examinerons en conclusion. Le premier axe s'attache à l'analyse des logiques médiatiques de production et de reproduction de la mémoire, et tend à mettre en évidence les règles qui président à la construction d'un consensus mémoriel (bien que les deux ne soient pas toujours nécessairement liés, puisque l'on peut aussi bien étudier les logiques de reproduction d'un conflit mémoriel, à moins de considérer que celles-ci ne participent à perpétuer une forme de consensus sur le conflit). Le second axe est consacré quant à lui aux logiques de transformation - celles qui font émerger de nouvelles voix ou qui font évoluer les modalités d'appréhension d'une mémoire - et éclaire davantage les dynamiques de concurrence mémorielle.

En ouverture de ce premier numéro thématique des Cahiers " Mémoire et Politique ", le texte de Marc VANESSE nous fait suivre au plus près, sur le mode du récit, le travail du journaliste de terrain en quête de témoignages sur la Deuxième Guerre mondiale, à l'occasion des commémorations de 2004 et 2005. Par l'anecdote tendre, il met en lumière la manière dont les conditions de travail et les pratiques professionnelles conditionnent la transmission médiatique de la mémoire, fruit d'un journalisme " au passé recomposé ». Loin d'une analyse déconnectée de ses objets, le texte fait voir la mémoire avant tout comme une expérience humaine, sensible, charnelle, dont la narration est à la fois la condition de sa transmission, de son partage, et le gage de sa fragilité. Ce faisant, il évoque le rapport du témoignage individuel à la mémoire collective, concluant que face à l'indicible, il reste au journaliste "cette voie étroite des parcours particuliers pour tenter de frôler l'universalité des tragédies humaines [...], dont le tableau final ressemble davantage à une œuvre impressionniste, cet art sublime de la décomposition du réel ».

\section{Consensus mémoriel et logiques de (re)production}

S'intéressant à l'influence du «mouvement des droits de l'Homme " $(\mathrm{MDH})$ dans la transition démocratique post-dictatoriale en Argentine, David COPELLO remet en question le statut accordé au Procès des Juntes dans cette évolution, à travers une analyse critique de la littérature savante locale. II met ainsi en évidence le processus de mythi- 
fication de l'événement, en examinant les logiques de circulation spécifiques au savoir académique en sciences sociales. L'analyse révèle les mécanismes de construction, de diffusion et de reproduction d'une mémoire positive et homogène du Procès des Juntes, assez éloignée de sa réception par les acteurs concernés à l'époque. Tout l'intérêt de l'article est de montrer comment, au départ d'une stratégie politique, a pu se construire, se perpétuer et s'imposer un consensus mémoriel autour d'un événement à l'origine très conflictuel, au travers d'un média particulier "de circulation plus restreinte [que les médias 'de masse'] mais aux effets très larges sur les schémas de perception, de périodisation et de mobilisation au présent de l'Histoire ».

Davantage concentrée sur les procédés textuels et intertextuels de construction mémorielle, la recherche de Virginie CERDEIRA porte sur un média hybride, à mi-chemin entre le livre d'histoire et le périodique d'information : Le Mercure François. Au lendemain de la mort d'Henri IV, cette publication poursuit clairement un objectif politique : assurer la continuité du régime de paix et éviter le retour des guerres civiles. L'auteure y analyse précisément les normes et pratiques d'écriture élaborant pour le présent et la postérité une mémoire unifiée, pacifiée, destinée à produire du consensus. En particulier, elle dissèque les procédés par lesquels le rédacteur mobilise différentes temporalités, articulées en « un mille-feuille mémoriel, monté à la fois par différentes couches de passé et par des mémoires plurielles que l'auteur unifie en une mémoire qu'il prescrit ». L'éthique de la vérité poursuivie par le Mercure ne s'identifie alors pas à une éthique de la neutralité, ce qui suppose que l'implication de l'auteur dans le texte les marqueurs d'énonciation - se pare d'une série de marqueurs d'authenticité et de véracité, notamment empruntés au genre historique.

L'article de Sébastien FEVRY envisage pour sa part l'articulation entre cinéma, politique et mémoire collective. Davantage encore, sans doute, que les films de la "vague sépia " qu'il étudie, c'est cette articulation elle-même qui constitue son objet. Considérant, dans le sillage de Stuart Hall, les productions médiatiques et culturelles comme un lieu où se rencontrent le discursif et le social, il compare les contenus mémoriels de la politique officielle du gouvernement Sarkozy à ceux du cinéma "sépia " qui a culminé sous sa présidence, pour les confronter ensuite à leur réception théorique. II montre alors comment des productions résonnantes du pouvoir et de la culture, véhiculant un même type de mémoire "centripète ", a-historique et fondée sur la valorisation d'un passé idéalisé, peuvent pourtant faire l'objet d'une réception différenciée en fonction des "cadres mémoriels chargés de l'exprimer ». Ainsi l'auteur réintroduit-il en quelque sorte la possibilité du conflit au sein de productions consensuelles, un même contenu mémoriel pouvant se trouver critiqué ou plébiscité selon qu'il apparaît 
dans le champ politique ou dans le champ culturel.

À la charnière des deux axes de ce numéro, le texte de Christoph BRÜLL et Catherine LANNEAU analyse le traitement médiatique de la recherche en histoire politique belge, révélant les mécanismes de reproduction d'un espace mémoriel divisé entre le nord et le sud du pays. La presse y est considérée comme une instance de réception de l'actualité de la recherche, manifestant une méconnaissance croissante, de la part de chaque communauté, de la mémoire collective de l'autre. Les auteurs mettent en évidence les conditions de production, de diffusion et de réception de la recherche sur la mémoire - notamment les facteurs professionnels, politiques, linguistiques ou socio-économiques - démontrant la manière dont journalistes, politiques et historiens contribuent à la mutation de l'État belge. Dégageant de cette façon les enjeux politiques et communautaires des rapports entre médias, mémoire et histoire, l'article montre in fine comment se perpétue un consensus sur la concurrence entre deux mémoires collectives en panne de dialogue.

\section{Concurrence mémorielle et logiques de transiormation}

Attachés à la question de la reconnaissance du génocide arménien, Joceline CHABOT et Richard GodIN s'intéressent ici au rôle tenu par les médias dans cette "guerre des mémoires " opposant les communautés turque et arménienne entre 1965 et 2005. Leur analyse de la presse canadienne éclaire les variations du récit médiatique dans le temps, au gré de l'actualité des événements et des prises de positions politiques d'individus ou de groupes d'intérêt dans le débat public. Distinguant les temporalités commémorative et événementielle, ils examinent notamment l'hypothèse selon laquelle la « mémoire médiatique " se construit en fonction des différentes "postures journalistiques présentes dans la structure du discours ». L'article met ainsi en évidence les logiques de transformation qui animent la construction de la « mémoire médiatique » : «En empruntant à l'une et à l'autre, [à la mémoire historique et à la mémoire communautaire], en résonnance à l'actualité des événements et des commémorations, la mémoire médiatique sédimente les faits constitués, mais ouvre aussi à de nouvelles reconstructions du passé ", qui ont pu aboutir ici à la reconnaissance médiatique du caractère génocidaire du drame arménien.

À côté des médias dominants, c'est aux "dispositifs mémoriels en ligne " que s'intéresse Sophie GEBEIL, et à la manière dont les acteurs s'en saisissent pour mener à bien des projets de valorisation de mémoires perçues comme marginalisées. Elle explore ainsi, à partir d'un corpus de sites web et d'entretiens avec leurs auteurs, l'évolution 
des modes d'expression des mémoires de l'immigration maghrébine sur l'internet français, en quête de reconnaissance et d'intégration à la mémoire collective nationale. Elle aborde notamment les enjeux de patrimonialisation - archivage et accessibilité des ressources numériques - et de politisation de ces mémoires minoritaires, menacées d'oubli et soumises dans le débat public à un « paradigme post-colonial " qui en conditionne la lecture. En particulier, l'article montre comment ces pratiques mémorielles sont intimement liées à des revendications d'ordre politique, découlant de l'intense politisation du débat sur l'immigration. Dans ce contexte, le web se présente comme un espace alternatif, privilégié pour l'expression de "contre-mémoires $\|^{5}$, dessinant finalement une "mosaïque de mémoires " dont la segmentation reste à interroger en regard des modes de «vivre ensemble » promus par le modèle républicain.

La contribution de Morane CHAVANON envisage également les médias en tant que ressource stratégique mobilisée par des acteurs mémoriels pris dans des "luttes patrimoniales ". Plus précisément, elle étudie la place et le rôle accordés aux médias dans les dossiers de candidature au " patrimoine culturel immatériel " $(\mathrm{PCl})$ de l'Unesco, dans une démarche qualitative d'inspiration sociologique. L'un des intérêts de l'article réside en ce qu'il met au jour un certain nombre de "qualités " prêtées aux médias par les acteurs patrimoniaux et, symétriquement, les griefs que ces derniers portent à leur encontre, les deux étant révélateurs de normes censées régir le rôle médiatique en matière patrimoniale, notamment relatives aux enjeux de transmission, de socialisation, d'identité communautaire. «À la fois instruments de légitimation des candidatures au $\mathrm{PCl}$, participant à la création performative d'une communauté imaginée et envisagés parmi les mesures de sauvegarde, les médias font également l'objet de revendications d'ordre social et politique ». Les médias sont alors le lieu par excellence où s'expriment, s'incarnent et se jouent les rapports de force à la base de l'attribution de valeur, déterminant la notion de "culture légitime $"$.

Le lecteur l'aura compris, "Médias en jeu, enjeux de mémoires » s'inscrit dans une perspective ouverte à plusieurs disciplines; principalement les sciences de l'information et de la communication, les sciences politiques, l'histoire, la sociologie et même la linguistique. Par ailleurs, de la diversité des médias abordés dans ce numéro se dégage d'emblée une perspective qui nous semble fructueuse pour les études sur la mémoire. Les médias, au sens large, constituent une face tout à la fois matérielle et sociale de la mémoire, qui s'y incarne, s'y négocie, 
s'y transmet, s'y oublie ou s'y dissout, bon gré, mal gré. Reflets autant que bâtisseurs, ils disent et font quelque chose du rapport de nos sociétés à leur passé, à leur identité et à leur "autre ", toujours (re) joué dans le présent. Ainsi la focale médiatique trace-t-elle un chemin par lequel la mémoire se laisse approcher, étudier, analyser sous son angle collectif et social.

Jenifer DEVRESSE

Assistante au Département des Arts et Sciences de la Communication (LEMME) de l'Université de Liège

\section{Pr. Dr Geoffrey GrandJEAN}

Chargé de cours au Département de science politique de l'Université de Liège

Titulaire du cours « Mémoire et politique " 